\title{
Capacity Optimisation Framework for Fast Charging Stations Operating under Cold Weather
}

\author{
I Safak Bayram \\ Department of Electronic and Electrical Engineering \\ University of Strathclyde \\ Glasgow, United Kingdom \\ safak.bayram@strath.ac.uk
}

\begin{abstract}
In this paper, we present a probabilistic capacity planning framework for electric vehicle (EV) fast charging stations that operate under cold weather. Existing literature on charging station modelling assumes that fast charging occurs at the rated capacity. However, recent empirical studies reveal that the actual charging rate depends on the battery and ambient temperatures and substantially reduces under cold weather. The proposed model is based on a multi-class queuing system where EV classes are determined based on temperature-dependent charger rates. The primary goal is to calculate minimum station capacity that can provide a certain level of quality of service (QoS) to each customer class. The performance metric describes the percentage of EVs that need to wait for service or leave the station. Case studies are provided to show the relationship between customer arrival rates, charging power and customer classes, and target QoS levels. The results further illustrate that the proposed framework achieves nearly one-third of capacity savings compared to baseline scenarios. The problems pertinent to temperature effects on $\mathrm{EV}$ charging require greater attention as EVs are becoming the main mode of transport in the next decade.
\end{abstract}

Index Terms-Electric Vehicles; Power Grid Impact; Battery Degradation; Cold weather;Queueing System

\section{INTRODUCTION}

Electric vehicles (EVs) are vital part of energy transformations towards net-zero future. Most developed countries have introduced policies to incentivise customers (e.g. tax credits, etc.) to purchase EVs [1], [2]. Such policies have been quite successful in several countries. For instance, new EV sales in Norway have outpaced petrol car sales in 2020, while more than 10 million EVs are expected to be on the road by 2030 in the UK as the government has introduced a ban on new petrol car sales [3]. Moreover, the US administration has announced to make trillion dollar investments to support EVs and electrify governmental vehicles [4].

Electrification of transport is a multifaceted transformational change that impacts both drivers and power grid operators. Drivers adopt new practices related to charging types and durations that are different from petrol filling stations. Power network operators, on the other hand, need to manage new challenges characterised by EVs at various levels such as accelerated transformer ageing [5] and power quality degradation (voltage dips, harmonics etc.) [6]. To that end, it is estimated by the National Grid in the UK that $£ 30$ billion of investments are required to support EV integration [7].
Existing studies on EV-grid integration assume that EVs operate under optimum driving temperatures (e.g. $\left(21.5^{\circ} \mathrm{C}\right)$ ) and can be charged at rated charger capacity. On the other hand, EV penetration is typically high in countries (e.g. Norway, UK, Canada) with cold ambient temperatures. In such countries, EV fast charging rate is restricted by the battery management units to avoid adverse effects on the battery cells [8]. According to a large-scale data driven analysis conducted in Norway, the average charge power is $40 \%$ less than the rated power of $50 \mathrm{~kW}$ and drivers charge considerably less than their battery capacity [9]. As shown in Fig.1, the charging power $(\mathrm{kW})$ and energy $(\mathrm{kWh})$ decrease in winter months and increases in summer season in line with mild temperatures. Similarly, the average charging sessions are inversely correlated with the charging power and less time is spent in warmer months.

Charging sessions under cold weather needs to be carefully investigated. For instance, the schedule of fleets (e.g. taxis, buses etc.) could be disturbed. Moreover, chargers that operate below rated capacity will introduce additional harmonics to the power network which will limit the maximum number of EVs that can be charged at the same time [10]. Low charging power will lead to over-provisioning of system resources at one location and necessitates more chargers at different sites to complement fast charger network. In addition, recent studies show that queueing times and reduced charger rates discourage EV drivers and nearly $20 \%$ of the EV owners in California switch back to petrol powered vehicles [11]. Therefore design of charging facilities is critical both for EV drivers and system operators. To that end, the contributions of this paper are as follows:

- We propose a stochastic charging station model which serves multi-class EV drivers. Customers are classified based on ambient temperature and associated charging power.

- We develop a capacity planning framework that computes minimum station capacity (in $\mathrm{kW}$ ) to accommodate $\mathrm{EV}$ customers of each type. Optimal station guarantees a certain level quality of service that is defined by loss of load probability (or probability of waiting for service or leaving the facility).

- A number of case studies are designed to provide insights 


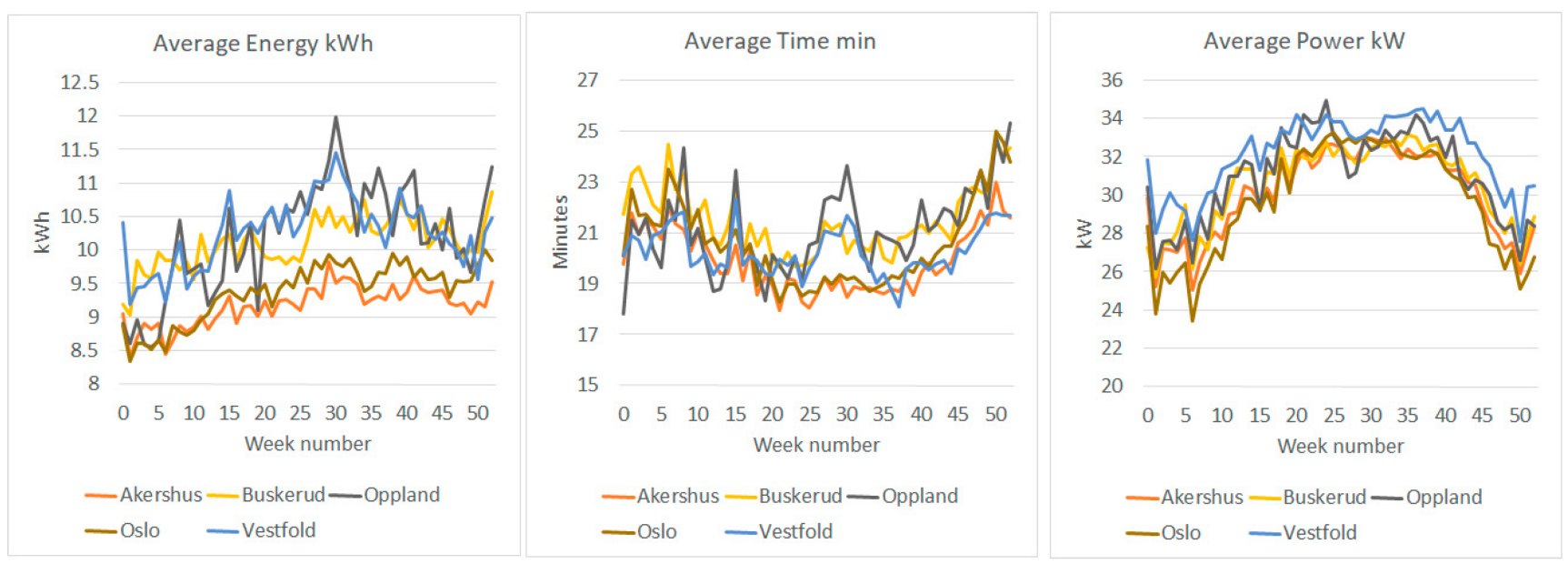

Fig. 1. Average energy charged, time used and average power of fast charging in Counties in Norway in 2017. Rated capacity of chargers are $50 \mathrm{~kW}$. Source: [9] (used with Creative Commons Attribution (CC BY) license) .

on different station parameters and how network operators can use the model to estimate EV load at a specific charging node. To the best of author's knowledge, this is the first study that considers ambient temperature impacts on charging station model.

\section{RELATED LITERATURE}

Over the last years, there has been a growing body of literature on the impacts of cold weather on EV charging and driving ranges. In [12] and [8], the driving range of EVs is studied and it was shown that significant percentage of battery energy is used for battery/cabin heating and to provide the extra traction needed to drive in winter. In [8], measurements were taken from Nissan Leaf which was driven in Winnipeg, Canada. The results show that the driving range drops from $162 \mathrm{~km}$ in $+28{ }^{\circ} \mathrm{C}$ to $44 \mathrm{~km}$ in $-26{ }^{\circ} \mathrm{C}$. In [13], researchers tested the fast charger efficiency under extreme temperatures. It was shown that the efficiency of most fast chargers reduces to $80-90 \%$ under $-15{ }^{\circ} \mathrm{C}$. In [14], authors investigated the impacts of $\mathrm{EV}$ charging under cold weather and present a probabilistic methodology to estimate the extra demand needed in winter months in the UK. It was shown that more than $500 \mathrm{MW}$ of extra load may be needed due to more often charging of vehicles. Authors further mentioned that the study does not involve EV fleets, therefore, actual extra demand could be significantly higher.

The literature on fast charging station modelling primarily involves queueing-based analysis [15]-[17]. In [15], authors proposed a performance evaluation framework for a fast charging station that is modelled as an $\mathrm{M} / \mathrm{M} / \mathrm{s}$ queue and customers are classified according to $\mathrm{AC}$ and $\mathrm{DC}$ charging types. They further developed a pricing-based scheduling policy to minimise EV waiting times. In [16], authors proposed a charging network operator who manages EV loads at spatially distributed charging stations to minimise waiting times. In the proposed model, each individual station is modelled as a

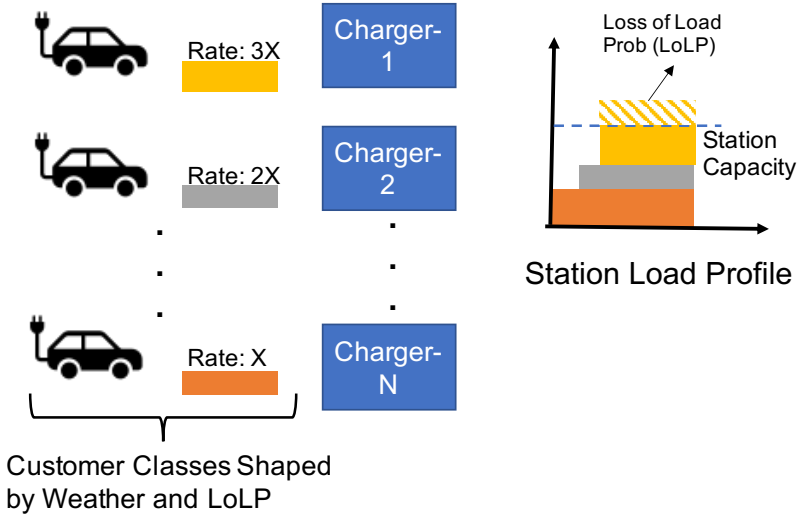

Fig. 2. Schematic overview of the proposed system.

queueing network. In [17], authors developed an optimisation model to calculate the capacity of a charging system equipped with solar panels and energy storage units. Different from the existing literature, the model presented in this paper employs a multi-rate Erlang-b system which captures the aggregate load profile of multi-class EVs based on their battery temperatures.

\section{PROPOSED FrAMEWORK}

We consider a DC fast charging station operating under cold weather. Even though the chargers are assumed to be identical, the rate of charging service is determined by the ambient and battery temperatures. More specifically, EVs with colder batteries are charged at lower rates (e.g. 20-30kW), while warmer batteries which have been in use for driving can be charged at faster rates (e.g. 45-50 kW). Hence, we consider $J$ distinct EV classes which are differentiated by the charging rate $b_{j}$ and loss of load probability (LoLP) target denoted by $\delta_{j}, j \in\{1, . ., J\}$. Note that the QoS metric LoLP for any customer class represents the probability that an arriving 
customer cannot receive service due to shortage of resources. To that end, our main interest is to calculate the minimum station capacity needed $C$ to enforce QoS guarantees for each customer type. Mathematically, the problem is formulated as

$$
C^{*}=\left\{\begin{array}{l}
\text { Minimise } \quad C \\
\text { Subject to } \quad \beta_{j} \leq \delta_{j}, \text { for } j \in\{1, \ldots, J\}
\end{array}\right.
$$

where $\beta_{j}$ is the LoLP probability for class $j$. Customer arrivals for each class $j$ are assumed to follow a Poisson process with rate $\lambda_{j}$, while the charging service durations are assumed to follow an exponential distribution with rate $\mu_{j}$. Note that such assumptions are very common in the literature as reported in [18]. An overview of the proposed system model is presented in Fig. 2. The charging station model described above falls under multi-dimensional loss systems (or multi-rate Erlang loss systems) where an arriving EV is either admitted to system or blocked due to lack of resources.

\section{A. Problem Formulation}

To model the capacity optimisation problem, we model the $\mathrm{EV}$ demand profile as a collection of $J$ independent queueing networks (see [19]). As a starting point, we assume that the power network resources are infinite. Let $Q_{\infty}^{j}$ represent the number of EVs of type $j$ with a charging rate of $b_{j}$ units of power (e.g. $50 \mathrm{~kW}$ under mild weather). Let $S$ be the total demand profile that is given by

$$
S=\sum_{j=1}^{J} b_{j} Q_{\infty}^{j}
$$

Due to Poisson arrival rates, the mean and the variance of $Q_{\infty}^{j}$ are given as $\mathbb{E}\left[Q_{\infty}^{j}\right]=\sigma^{2}\left[Q_{\infty}^{j}\right]=q_{j}=\frac{\lambda_{j}}{\mu_{j}}$. Therefore

$$
\mathbb{E}[S]=\sum_{j=1}^{J} b_{j} q_{j}, \text { and } \sigma^{2}[S]=\sum_{j=1}^{J} b_{j}^{2} q_{j} .
$$

Now, by considering finite station capacity represented by $C$, the carried load system defined by $Q_{C}^{j}$ is defined as the number of simultaneous EVs at the charging station. Then, as described previously, the loss of load even occurs when an EV of type $j$ finds available resources less than $C-b_{j}$. For a given set of EV charging rates $\boldsymbol{b}=\left[b_{1}, \ldots, b_{J}\right]$ and mean number of arrival rates $\boldsymbol{q}=\left[q_{1}, \ldots, q_{J}\right]$ (used interchangeable with $\lambda_{j}$ for ease of presentation), LoLP for customer type $j$ is denoted by $\beta_{j} \in\{0,1\}$ and mathematically written as

$\beta_{j}(\boldsymbol{q}, \boldsymbol{b})=\mathrm{P}\left\{C-b_{j}<\sum_{j=1}^{J} b_{j} Q_{\infty}^{j} \leq C \mid \sum_{j=1}^{J} b_{j} Q_{\infty}^{j} \leq C\right\}$

$$
=\frac{\mathrm{P}\left\{C-b_{j}<\sum_{j=1}^{J} b_{j} Q_{\infty}^{j} \leq C\right\}}{\mathrm{P}\left\{\sum_{j=1}^{J} b_{j} Q_{\infty}^{j} \leq C\right\}} .
$$

In order for station capacity to provide individual QoS targets $\left\{\delta_{j}\right\}$, optimal capacity $C^{*}$ needs to be as big as the mean offered load given in (2). Due to probabilistic nature of the EV arrivals, additional capacity is needed to provide demand variations. To that end, $C^{*}$ is set equal to expected load $\mathbb{E}[S]$ and adjusted by an extra term that is proportional to its variance denoted by $x \cdot \sigma^{2}[S]$. To find a closed form expression, the system is scaled with $\varsigma>0$ to devise the following expression for the system capacity

$$
\bar{C}(\varsigma, x)=\varsigma \sum_{j=1}^{J} b_{j} q_{j}+x \sqrt{\varsigma \sum_{j=1}^{J} b_{j}^{2} q_{j}} .
$$

Using the limiting relations described [20], we have

$$
\lim _{\varsigma \rightarrow \infty} \sqrt{\varsigma} \beta_{j}(\boldsymbol{q}, \boldsymbol{b})=\frac{b_{j}}{\sqrt{\sum_{j=1}^{J} b_{j}^{2} q_{j}}} \cdot \frac{\phi(x)}{\varphi(x)}
$$

where $\phi(x)=\frac{1}{\sqrt{2 \pi}} e^{-x^{2} / 2}$ and $\varphi(x)=\frac{1}{\sqrt{2 \pi}} \int_{-\infty}^{x} e^{-t^{2} / 2} d t$. We further define function $\psi$ as the inverse of $\frac{\phi}{\varphi}$ for $\forall x$, that is:

$$
\frac{\phi(\psi(x))}{\varphi(\psi(x))}=x
$$

Notice that $\psi(\cdot)$ is a strictly decreasing function with $\psi(y)+$ $y>0, \forall y$ ( [20]). Therefore, the asymptotic behavior of the QoS constraint $\beta^{j}(\boldsymbol{q}, \boldsymbol{b}) \leq \delta_{j}$ can be written as:

$$
x \geq \psi\left(\frac{\delta_{j}}{b_{j}} \sqrt{\sum_{j=1}^{J} b_{j}^{2} q_{j}}\right) .
$$

The station capacity should further provide QoS targets. Hence, the inequality in (9) yields

$$
x \geq \psi\left(\min _{1 \leq j \leq J}\left\{\frac{\delta_{j}}{b_{j}} \sqrt{\sum_{j=1}^{J} b_{j}^{2} q_{j}}\right\}\right) .
$$

The minimum station capacity can be found by solving the provisioning problem in (1) is

$$
C^{*}=\sum_{j=1}^{J} b_{j} \frac{\lambda_{j}}{\mu_{j}}+\psi\left(\min _{1 \leq j \leq J} \frac{\delta_{j}}{b_{j}} \sqrt{\sum_{j=1}^{J} b_{j}^{2} \lambda_{j}}\right) \sqrt{\sum_{j=1}^{J} b_{j}^{2} \lambda_{j}},
$$

where $\psi(\cdot)$ can be numerically computed by solving [21]:

$$
x^{-1} e^{-0.5 \psi(x)^{2}}-\sqrt{2 \pi} \operatorname{erf}\left(\frac{1}{\sqrt{2}} \psi(x)\right)-x \sqrt{0.5 \pi}=0 \text {. }
$$

Note that equations (11) and (12) are the main results to compute optimal station capacity. The only missing terms are LoLP probabilities represented by $\delta_{j}$ and associated computational methods are presented in the next section.

\section{B. Loss-of-Load-Probability Computation Methods}

To compute the LLoP functions, we need to analyse $J$ independent Markov chains in which system state is defined as the number of EVs of the same time, i.e. $Q \triangleq\left[Q_{C}^{1}, \ldots, Q_{C}^{J}\right]$ and the state space is denoted by $\Omega \triangleq\left\{\boldsymbol{Q}: \sum_{j=1}^{J} b_{j} Q_{\infty}^{j} \leq C\right\}$. Let $\tilde{Q}_{C}^{j}$ represent the maximum number of EVs of type $j$ 


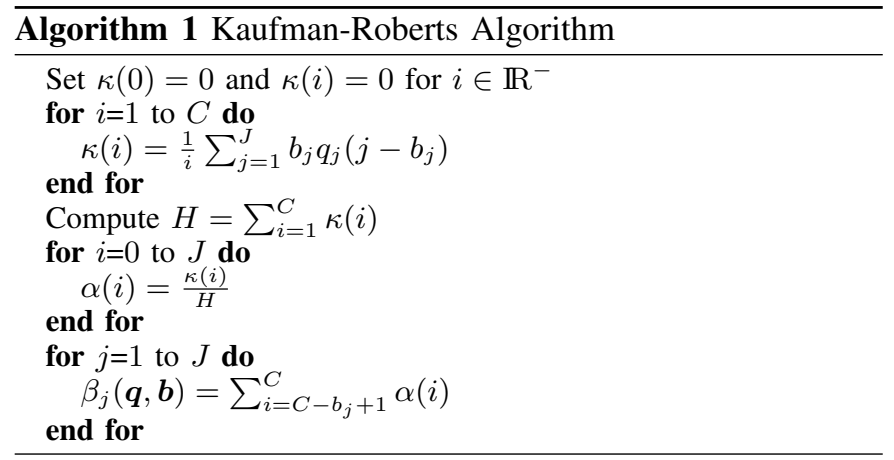

that can be charged simultaneously. Assuming that the charger rates are ordered $b_{1} \geq \cdots \geq b_{J} \geq 0$, the following can be written $0 \leq \tilde{Q}_{C}^{1} \leq \cdots \leq \tilde{Q}_{C}^{J}$. Then the probability of being at state $\boldsymbol{Q}$ is [19] $\pi(\mathbf{Q})=\prod_{j=1}^{J} \frac{q_{j}^{Q_{\infty}^{j}}}{Q_{\infty}^{j} !} e^{-q_{j}}$. By conditioning on the finite capacity, and a generic state $Q$ probability distribution is computed as:

$$
\pi(\boldsymbol{Q})=\frac{\bar{\pi}(\mathbf{Q})}{\sum_{\tilde{Q} \in \Omega} \bar{\pi}(\tilde{\mathbf{Q}})} .
$$

The blocking states for customer type $j$ is written as

$$
\Psi_{j}=\left\{\boldsymbol{Q}: C-b_{j}<\sum_{k=1}^{J} b_{k} Q_{C}^{k} \leq C\right\} .
$$

Moreover, the LoLP function can be written as

$$
\beta^{j}(\boldsymbol{q}, \boldsymbol{b})=\sum_{s \in \Psi_{j}} \pi(s)=1-\sum_{s \notin \Psi_{j}} \pi(s),
$$

Moreover, the function $H(C, J)$ is defined as

$$
H(C, J) \triangleq \sum_{\{\boldsymbol{Q}: \boldsymbol{b} \boldsymbol{Q} \leq C\}} \prod_{j=1}^{J} \frac{q_{j}^{Q^{j}}}{Q^{j !}},
$$

which is used to compute the LoLP for class $j$ explicitly as:

$$
\beta_{j}(\boldsymbol{q}, \boldsymbol{b})=1-\frac{H\left(C-b_{j}, J\right)}{H(C, J)} .
$$

Using the defined functions, we use recursive methods described by Kaufman-Roberts algorithms (shown in Algorithm 1) [19]. Let $c$ denote the real-time load profile of the station, then we have

$$
\alpha(c)=\sum_{\{\boldsymbol{Q}: \boldsymbol{b} \boldsymbol{Q} \leq c\}} \frac{q_{j}^{Q^{j}}}{Q^{j}} \cdot \frac{1}{H(C, J)},
$$

and the LoLP for type $j$ can be calculated by using solving the following equation:

$$
\beta_{j}(\boldsymbol{q}, \boldsymbol{b})=\sum_{i=0}^{b_{j}-1} \alpha(C-i) .
$$

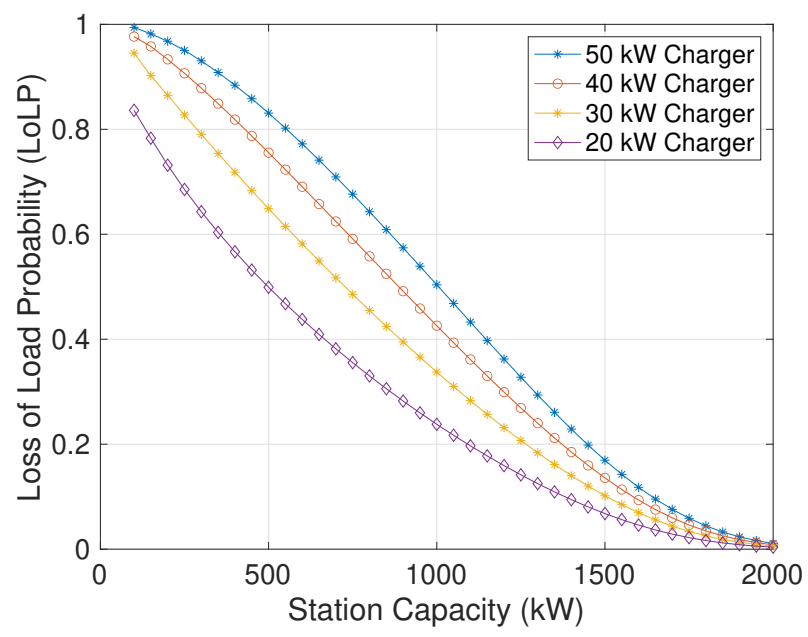

Fig. 3. Loss of load probability for four classes and fixed arrival rate of $\lambda=50$.

\section{NumericAl EVAluATIONS}

To provide more insights on the proposed framework, the following case studies are performed. It is assumed that the charging station serves four different $(J=4) \mathrm{EV}$ classes that are classified based on the battery temperature and charging power. By assuming the fast charging occurs at $b_{1}=50$ $\mathrm{kW}$, the other charging rates are determined as $b_{2}=40 \mathrm{~kW}$, $b_{3}=30 \mathrm{~kW}$, and $b_{4}=20 \mathrm{~kW}$. The charger rates are estimated based on the measurements provided in [22] (see Fig. 2 of the reference). For instance, under zero celsius it takes about 60 minutes to reach $80 \%$ SoC level of a Nissan Leaf (24 $\mathrm{kWh}$ ). On the other hand, it only takes 20-25 minutes to reach the same SoC level under 25-30 Celsius. Moreover, by considering most popular EV battery sizes and $20 \%$ to $80 \%$ SoC charging habits, it is assumed that on average each vehicle wants to transfer $30 \mathrm{kWh}$ of energy. The overview of the customer classes are given in Table I.

In Fig. 3, the LoLP for each charger class is evaluated for the aforementioned four charger classes and each arrival rate is assumed to be $\lambda_{j \in 1,2,3,4}=12.5$. It can be observed that as the station capacity increases the LoLP approaches to zero. Moreover, the LoLP for class $4(20 \mathrm{~kW})$ is strickly less than the others as the charger rate $b_{4}$ is less than the others $b_{4}<$ $b_{1,2,3}$. Therefore, the most dominant factor in determining the station capacity respect to QoS targets is the customer class with the highest charging rate.

Second evaluation case study is evaluated for a wide range system parameters. Class-specific arrival rates are assumed to be equal to each other $\left(\lambda_{1}=\lambda_{2}=\lambda_{3}=\lambda_{4}\right)$ and total arrival rate is varied from 10 to 20 (EVs per hour). The target QoS levels for each customer class is presented in Table II. Four different case studies are presented in Fig. 4. Case (a) has the most stringent LoLP targets as each EV class is expected to receive service under 0.01 QoS levels. Therefore, the station capacity takes the highest value for case (a). In a similar 

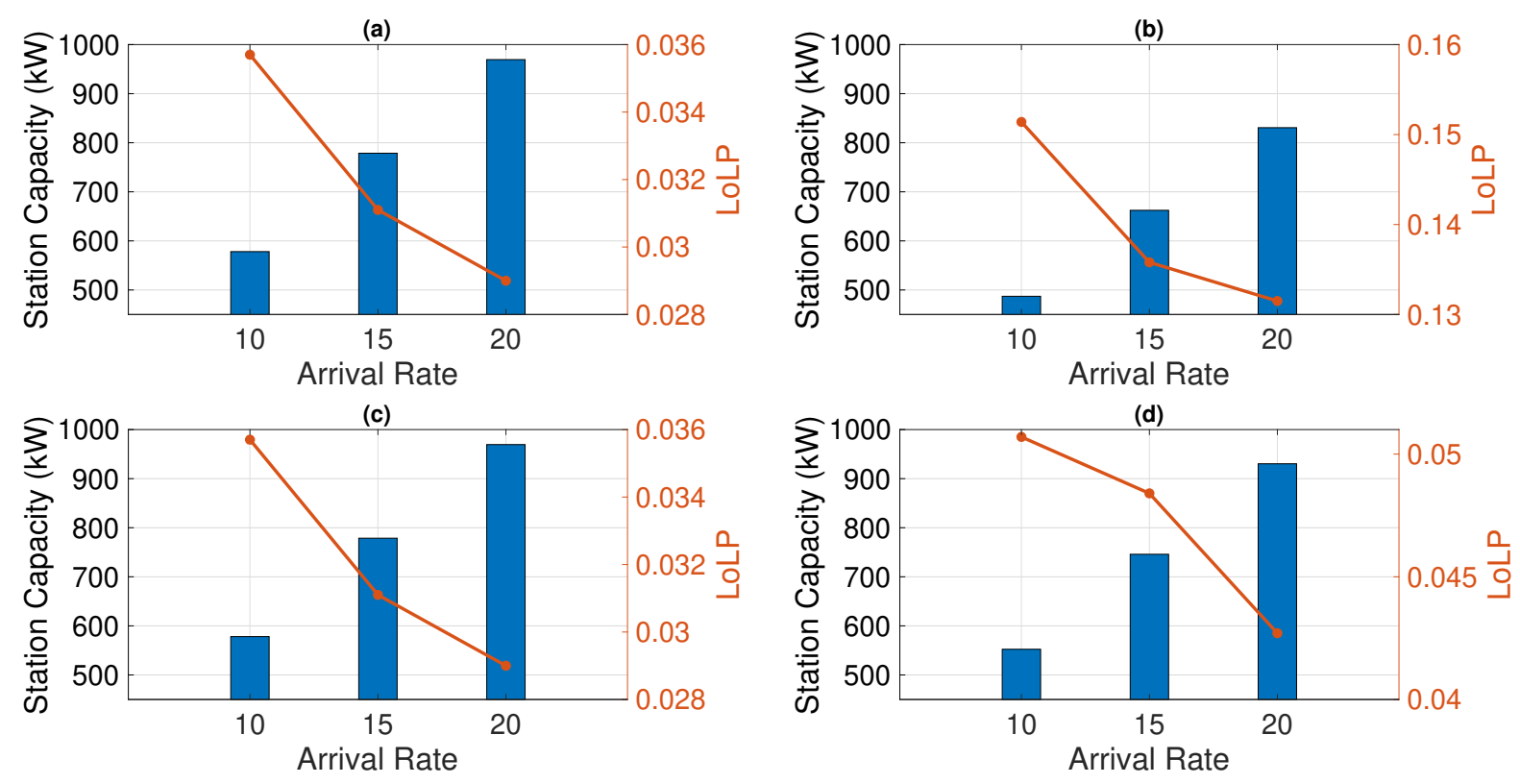

Fig. 4. Numerical evaluations for station capacity. Target LoLP values are presented in Table II. Right y-axis shows sum of actual LoLP levels.

TABLE I

CUSTOMER CLASSES FOR THE CASE STUDIES.

\begin{tabular}{lccc}
\hline Class & $\begin{array}{c}\text { Charge } \\
\text { Rate }(\mathrm{kW})\end{array}$ & $\begin{array}{c}\text { Average } \\
\text { Duration (min) }\end{array}$ & $\begin{array}{c}\text { Service Rate } \\
\text { (no of EVs per hour) }\end{array}$ \\
\hline 1 & 50 & 36 & 1.67 \\
2 & 40 & 45 & 1.33 \\
3 & 30 & 60 & 1 \\
4 & 20 & 90 & 0.66 \\
\hline
\end{tabular}

TABLE II

TARGET LOLP FOR CASES (A)-(D) IN FIG. 4

\begin{tabular}{lllll}
\hline & \multicolumn{4}{c}{ Target LoLP $\left(\delta_{j}\right)$} \\
Case & $\delta_{1}$ & $\delta_{2}$ & $\delta_{3}$ & $\delta_{4}$ \\
\hline $\mathrm{a}$ & 0.01 & 0.01 & 0.01 & 0.01 \\
$\mathrm{~b}$ & 0.05 & 0.05 & 0.05 & 0.05 \\
$\mathrm{c}$ & 0.01 & 0.01 & 0.05 & 0.05 \\
$\mathrm{~d}$ & 0.05 & 0.05 & 0.01 & 0.01 \\
\hline
\end{tabular}

manner, the lowest station capacity is required for case (b) as each target LoLP is set to $\delta_{1,2,3,4}=0.05$. When cases (c) and (d) are compared, higher capacity is required at case (c) as EV classes $1(50 \mathrm{~kW})$ and $2(40 \mathrm{~kW})$ requires more resources than classes $3(30 \mathrm{~kW})$ and $4(20 \mathrm{~kW})$.

To capture the weight of different customer classes, another case study is presented. In this case, the station is assumed to operate in a colder climate and the most EVs are with a colder battery. More specifically, customers demanding $50 \mathrm{~kW}$ and $40 \mathrm{~kW}$ chargers represent $1 / 8$ of the customers, while remaining customers demand $30 \mathrm{~kW}$ and $20 \mathrm{~kW}$ chargers. Similar to the case in Fig. 4 (c), the LoLP probabilities are set as $\delta_{1}=0.01, \delta_{2}=0.01, \delta_{3}=0.05$, and $\delta_{4}=0.05$.

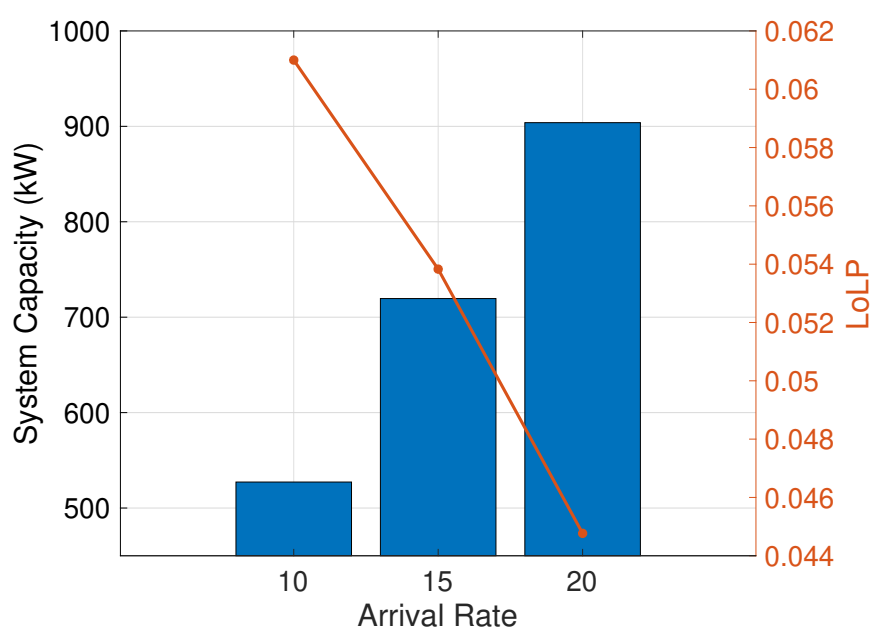

Fig. 5. Numerical evaluation for different customer portfolio $(1 / 8$ of EVs request $50 \mathrm{~kW}$ and $40 \mathrm{~kW}$, while the rest of the customer are class 3 and 4 . Y-axis depicts total LoLP for all classes.).

When compared to case (c), the system capacity significantly reduces because the share of customers in the first two classes reduce. This case study confirms the main finding presented in (11) that the capacity is related to the multiplication of arrival and charger rates. As a final evaluation, the capacity savings are calculated with respect to a baseline scenario where each customer class requires a LoLP guarantees close to zero $\left(\delta_{1,2,3,4}=0.0001\right)$. As shown in Fig. 6 , as the arrival rate increases, the savings decrease due to increased system utilisation. Moreover, for high LoLP targets $\left(\delta_{j}=005, \forall j\right)$, the savings are higher as more EVs are allowed to wait for 


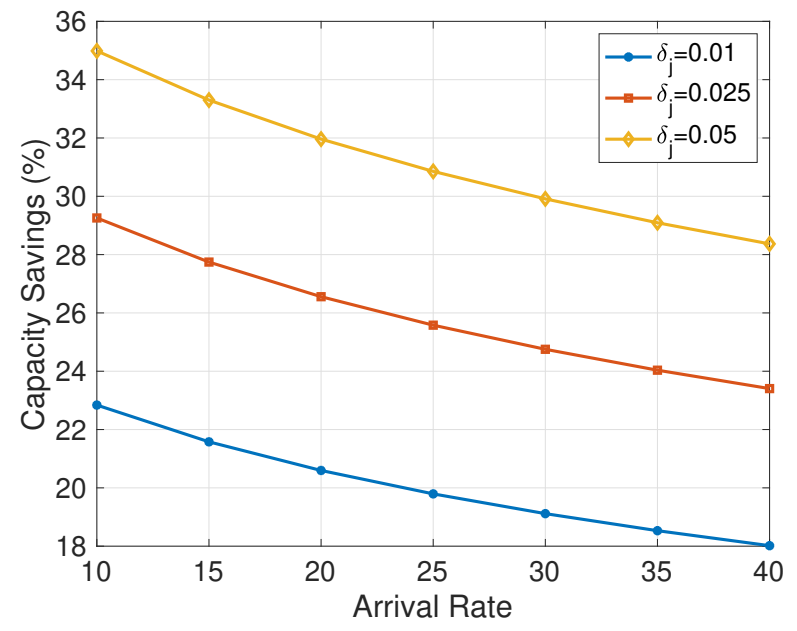

Fig. 6. Capacity savings with respect to baseline scenario $\left(\delta_{1,2,3,4}=0.001\right)$.

service.

\section{CONCLUSIONS}

This paper presented a stochastic capacity planning framework for fast EV charging stations operating under cold weather. Through existing empirical studies, it was shown that low ambient temperatures limit the battery charging rates, hence, EVs arriving to a charging station are naturally segmented into multiple classes. In public facilities due to limited space, the charging station capacity was determined to minimise the probability of waiting for each customer type. Therefore, loss of load probability (LoLP) was used to evaluate the system performance. LoLP is the probability that the aggregate system demand exceeds station capacity, hence, the new EV arrivals are either need to wait or leave the system. The capacity planning model is further linked to guarantee QoS levels defined as the maximum LoLP level for each customer type. A number of case studies were performance to investigate the interplay between customer statistics, LoLP levels and station capacity. The results show that the stations will be less loaded in winter months due to limited charger rates.

\section{REFERENCES}

[1] S. Pye, F. G. Li, J. Price, and B. Fais, "Achieving net-zero emission through the reframing of uk national targets in the post-paris agreement era," Nature Energy, vol. 2, no. 3, pp. 1-7, 2017.

[2] B. K. Sovacool, L. Noel, J. Kester, and G. Z. de Rubens, "Reviewing nordic transport challenges and climate policy priorities: Expert perceptions of decarbonisation in denmark, finland, iceland, norway, sweden,' Energy, vol. 165, pp. 532-542, 2018.

[3] N. Rietmann, B. Hügler, and T. Lieven, "Forecasting the trajectory of electric vehicle sales and the consequences for worldwide co2 emissions," Journal of Cleaner Production, vol. 261, p. 121038, 2020.

[4] "4 recommendations to electrify the us federal fleet." [Online] Available: https:/www.wri.org/insights/4-recommendations-electrifyus-federal-fleet

[5] C. d. M. Affonso and M. Kezunovic, "Probabilisitic sizing of pv generation on commercial parking lot with pevs to avoid transformer aging," in 2019 IEEE Milan PowerTech, June 2019, pp. 1-6.
[6] K. Clement-Nyns, E. Haesen, and J. Driesen, "The impact of charging plug-in hybrid electric vehicles on a residential distribution grid," IEEE Transactions on power systems, vol. 25, no. 1, pp. 371-380, 2009.

[7] "Electric vehicle strategy, scottish and souther electricity networks." [Online]. Available: http://news.ssen.co.uk/news/allarticles/2020/march/ssen-publishes-electric-vehicle-strategy-withcustomers-and-stakeholders-at-its-heart/

[8] J. R. M. D. Reyes, R. V. Parsons, and R. Hoemsen, "Winter happens: The effect of ambient temperature on the travel range of electric vehicles," IEEE Transactions on Vehicular Technology, vol. 65, no. 6, pp. 4016-4022, 2016.

[9] E. Figenbaum, "Battery electric vehicle fast charging-evidence from the norwegian market," World Electric Vehicle Journal, vol. 11, no. 2, p. 38, 2020.

[10] R. Pogaki, "Western power distribution electric vehicle charging (pse0564001)," Tech. Rep., 2018.

[11] S. Hardman and G. Tal, "Understanding discontinuance among california's electric vehicle owners," Nature Energy, vol. 6, no. 5, pp. 538-545, 2021.

[12] D. Ramsey, A. Bouscayrol, L. Boulon, and A. Vaudrey, "Simulation of an electric vehicle to study the impact of cabin heating on the driving range," in 2020 IEEE 91st Vehicular Technology Conference (VTC2020Spring). IEEE, 2020, pp. 1-5.

[13] G. Trentadue, A. Lucas, M. Otura, K. Pliakostathis, M. Zanni, and H. Scholz, "Evaluation of fast charging efficiency under extreme temperatures," Energies, vol. 11, no. 8, p. 1937, 2018.

[14] I. Koncar, , and I. S. Bayram, "A probabilistic methodology to quantify the impacts of cold weather on electric vehicle demand: A case study in the uk," IEEE Access, 2021.

[15] I. Zenginis, J. Vardakas, N. Zorba, and C. Verikoukis, "Performance evaluation of a multi-standard fast charging station for electric vehicles," IEEE transactions on smart grid, vol. 9, no. 5, pp. 4480-4489, 2017.

[16] A. Moradipari and M. Alizadeh, "Pricing and routing mechanisms for differentiated services in an electric vehicle public charging station network," IEEE Transactions on Smart Grid, vol. 11, no. 2, pp. 14891499, 2019.

[17] J. Ugirumurera and Z. J. Haas, "Optimal capacity sizing for completely green charging systems for electric vehicles," IEEE Transactions on Transportation Electrification, vol. 3, no. 3, pp. 565-577, 2017.

[18] I. S. Bayram, M. Devetsikiotis, and R. Jovanovic, "Optimal design of electric vehicle charging stations for commercial premises," International Journal of Energy Research, vol. n/a, no. n/a.

[19] I. S. Bayram, A. Tajer, M. Abdallah, and K. Qaraqe, "Capacity planning frameworks for electric vehicle charging stations with multiclass customers," IEEE Transactions on Smart Grid, vol. 6, no. 4, pp. 1934 1943, 2015.

[20] D. Mitra and J. Morrison, "Erlang capacity and uniform approximations for shared unbuffered resources," IEEE/ACM Transactions on Networking, vol. 2, no. 6, pp. 558-570, Dec 1994.

[21] W. Tian and H. Perros, "Analysis and provisioning of a circuit-switched link with variable-demand customers," in Managing Traffic Performance in Converged Networks. Springer, 2007, pp. 890-900.

[22] Y. Motoaki, W. Yi, and S. Salisbury, "Empirical analysis of electric vehicle fast charging under cold temperatures," Energy Policy, vol. 122, pp. 162-168, 2018. 